\title{
Discrete elastic model for stretching-induced flagellar polymorphs
}

\author{
H. WADA ${ }^{1,2}$ and R. R. NETZ ${ }^{1}$ \\ Physics Department, Technical University Munich, 85748 Garching, Germany \\ Yukawa Institute for Theoretical Physics, Kyoto Universiy, Kyoto, 606-8502, Japan
}

PACS 87.16.Ka - Filaments, microtubules, their networks, and supermolecular assemblies

PACS 87.15. - - - Biomolecules; structure and physical properties

PACS 64.70.Nd - Structural transitions in nanoscale materials

\begin{abstract}
Force-induced reversible transformations between coiled and normal polymorphs of bacterial flagella have been observed in recent optical-tweezer experiment. We introduce a discrete elastic rod model with two competing helical states governed by a fluctuating spin-like variable that represents the underlying conformational states of flagellin monomers. Using hybrid Brownian dynamics Monte-Carlo simulations, we show that a helix undergoes shape transitions dominated by domain wall nucleation and motion in response to externally applied uniaxial tension. A scaling argument for the critical force is presented in good agreement with experimental and simulation results. Stretching rate-dependent elasticity including a buckling instability are found, also consistent with the experiment.
\end{abstract}

Introduction - Many motile bacteria achieve directed propulsion in a viscous environment by rotating flagellar filaments [1]. A number of common bacteria, such as Escherichia coli or Salmonella, are externally flagellated, where each flagellum consists of a helically-shaped elastic filament that is attached to a rotary motor embedded in the rigid cell wall via a flexible hook. The flagellar filament is a large homogeneous assembly of a single protein, flagellin, but transforms between several helical shapes of different pitch, radius and handedness in response to environmental or mechanical stimuli such as change of temperature, $\mathrm{pH}$, or externally applied forces and torques [2]. For example, Salmonella swim by rotating their left-handed helical filaments counter-clockwise, forming a co-ordinated bundle of filaments $[3,4]$. The reverse of the motor rotational direction gives rise to a tumbling motion of the cell body, often (but not always) accompanied by changes in filament chirality to right-handed $[2,5]$. By regulating the relative durations of swimming and tumbling modes, bacteria are able to migrate through a stimulus gradient in the favorable direction.

The flagellar filament is composed of eleven protofilaments that each consist of a stack of flagellin monomers and that are wrapped around each other [6]. As first proposed by Asakura [7] and later proved by crystallography [8], a flagellin monomer can take two conformations, L-type and R-type, that are slightly different in intrinsic twist and length. Each protofilament can be assumed to consist entirely of either L or R monomers, however, the complete filament is thought to contain variable numbers of L and R-type protofilaments. Depending on how many protofilaments are of the $\mathrm{L}$ or $\mathrm{R}$ type, the flagellum takes different polymorphic helical states, thereby minimizing the elastic strain energy. A simple geometric model developed by Calladine in line with this idea [9] explained the observed spectrum of flagellar polymorphic forms. A coarse-grained continuum rod model has also been developed recently [10].

The Asakura-Calladine model has revealed the design principle of bacterial flagellar filament. Recently, dynamics of flagellar polymorphic transitions have moved into the focus. Turner et al. have reported an intricate sequence of transformations of fluorescently labelled $E$. coli flagella during a single run-to-tumble mode switching [11]. Clearly, understanding elastic energy barriers between polymorphic forms is crucial for the understanding of switching dynamics. Several studies, both experimental and theoretical, have addressed this issue $[2,12-14]$. Hotani reported cyclic chirality transformations of Salmonella flagella tethered at one end to a glass surface and subjected to an external fluid flow [12]. Above a certain critical flow velocity (a few $\mu \mathrm{m} / \mathrm{s}$ ), chirality flips occur at the tethered end and nucleated chirality domains grow and propagate steadily down the filament. The magnitudes of pulling force or mechanical torque necessary for the onset of the normal-to-semi-coiled 
transition were estimated as $15 \mathrm{pN}$ and $10^{-18} \mathrm{~N}$ m respectively, based on a slender-body hydrodynamics analysis of the experimental geometry. Coombs and Goldstein [14] theoretically analysed and rationalized Hotani's observation based on a bistable helix model introduced earlier by Goldstein and co-workers [15]. Using an optical tweezer setup, Darnton and Berg have recently measured the forceextension curves of Salmonella flagella filaments [16]. Mechanical stretching induces a reversible polymorphic transformation between coiled and normal state at a threshold force around 3-5 pN, characterized by large hysteresis during pulling and relaxing. The measured force is shown to be strongly rate-dependent, suggesting that the observed polymorphic transformation is kinetically controlled. Stretching experiments of Salmonella flagella have also been performed using atomic force microscopy by Cluzel et al. [17]. Since atomistic simulations are impossible with present computer power due to the huge size of a flagellum and the large time scales involved, coarsegraining is imperative. In one recent simulation study, a flagellin monomer is represented by 15 point masses with interactions parameterized based on the known protein crystal structure [18]. Using an implicit solvent model, different helical structures are observed depending on rotational direction and in qualitative accord with experiments. But still, the numerical simulation effort only allows to simulate the model flagellum for about $30 \mu$ s which is short compared to intrinsic time scales of polymorphic dynamics [18]. It is therefore desirable to take the coarsegraining to an even higher level.

In this paper, we introduce an elastic helix model that is coupled to an internal discrete variable that locally describes polymorphic states. In the present formulation we consider an Ising-like spin variable that switches between two states of different helix pitch and radius. We introduce a hybrid Brownian-dynamics Monte-Carlo simulation and show that our model reproduces well the observed forceextension relationship of flagellar filaments accompanying polymorphic transformations [16]. As in experiments, the force-extension curves are strongly stretching-rate dependent. We also give simple scaling arguments for the critical switching force, in good agreement with experimental and our numerical data.

Model - Within linear elasticity theory, the bending and twisting energy for an isotropic helical rod of contour length $L$ parameterized by arclength $s$ is given by

$$
E_{e l}=\frac{1}{2} \int_{0}^{L} d s\left[A \Omega_{1}^{2}+A\left(\Omega_{2}-\Omega_{2}^{0}\right)^{2}+C\left(\Omega_{3}-\Omega_{3}^{0}\right)^{2}\right],
$$

where $A$ and $C$ stand, respectively, for the bending and twisting modulus [19]. $\boldsymbol{\Omega}=\left(\Omega_{1}, \Omega_{2}, \Omega_{3}\right)$ is the strain rate vector; the curvature $\kappa$ satisfies $\kappa^{2}=\Omega_{1}^{2}+\Omega_{2}^{2}$ and $\Omega_{3}$ is the twist density. The ground-state shape of a filament is specified by the intrinsic curvature $\kappa_{0}$ and torsion $\tau_{0}$, related to the radius $R$ and pitch $P$ via $\kappa_{0}=$ $4 \pi^{2} R /\left(P^{2}+4 \pi^{2} R^{2}\right)$ and $\tau_{0}=2 \pi P /\left(P^{2}+4 \pi^{2} R^{2}\right)$. Al- ternatively, one can specify the pitch angle $\psi$ through $\tan \psi=2 \pi R / P$, and the contour length of a single helical turn, $\ell=\left(P^{2}+4 \pi^{2} R^{2}\right)^{1 / 2}$. The relation between these two descriptions is given by $\kappa_{0}=(2 \pi / \ell) \sin \psi$ and $\tau_{0}=(2 \pi / \ell) \cos \psi$. Experimentally, the so-called "coiled" state of a filament is the equilibrium shape at $\mathrm{pH} 4$ and $3^{\circ} \mathrm{C}$ (see the phase diagram in ref. [16]). We in particular study the polymorphic transition from the coiled to the so-called "normal" state. For the coiled and normal forms of Salmonella, which we respectively label as 1 and $2, \ell$ and $\psi$ were measured as $\left(\ell_{1}, \ell_{2}\right)=(3.4 \mu \mathrm{m}, 2.5 \mu \mathrm{m})$ and $\left(\psi_{1}, \psi_{2}\right)=\left(76.7^{\circ}, 31.3^{\circ}\right)$ [16]. In the present study, we for simplicity set the length $\ell$ for both forms equal and choose pitch angles $\psi_{1}=73.3^{\circ}$ and $\psi_{2}=29.7^{\circ}$, close to the experimental values. The intrinsic curvature and torsion for each helical state follow as $\kappa_{0 m}=(2 \pi / \ell) \sin \psi_{m}$ and $\tau_{0 m}=(2 \pi / \ell) \cos \psi_{m}$, where $m=1,2$.

In the simulations we consider a chain of $N+1$ connected spheres of diameter $a$. Each bead is specified by its position $\mathbf{r}_{j}$ and rotation angle about the local tangent $\phi_{j}$, from which the discrete strain rate $\boldsymbol{\Omega}_{j}$ is calculated. Analogous to the helix-coil model for biopolymers [20], each sphere carries a discrete variable $\sigma_{j}$, where $\sigma_{j}=1$ specifies the coiled form and $\sigma_{j}=-1$ the normal form. To account for cooperativity, induced by elastic strain at the interface between two polymorphic states, a short-range interaction $J$ between neighbouring "spin" variables $\sigma_{j}$ and $\sigma_{j+1}$ is included. The total energy functional of the system (apart from a stretching contribution introduced later) reads in discretized form

$$
\begin{aligned}
& E[\boldsymbol{\Omega}, \sigma]=-J \sum_{\langle i, j\rangle} \sigma_{i} \sigma_{j}-h a \sum_{j=1}^{N} \sigma_{j} \\
& +\frac{a}{2} \sum_{j=1}^{N}\left[A \Omega_{1 j}^{2}+A\left(\Omega_{2 j}-\Omega_{2 j}^{0}\right)^{2}+C\left(\Omega_{3 j}-\Omega_{3 j}^{0}\right)^{2}\right]
\end{aligned}
$$

where $\langle i, j\rangle$ implies summation over nearest neighbors and the $\sigma$-dependent intrinsic strain rates are $\Omega_{2 j}^{0}=\sigma_{j}\left(\kappa_{01}-\right.$ $\left.\kappa_{02}\right) / 2+\left(\kappa_{01}+\kappa_{02}\right) / 2$, and $\Omega_{3 j}^{0}=\sigma_{j}\left(\tau_{01}-\tau_{02}\right) / 2+\left(\tau_{01}+\right.$ $\left.\tau_{02}\right) / 2$. The chemical potential bias per length, $h$, accounts for a preference of one state over the other, and depends on environmental conditions such as solution $\mathrm{pH}$ or salinity.

Effective potential - To gain insight into the coupling between helix elasticity and the polymorphic degrees of freedom, we formally define an effective elastic energy density $f(\boldsymbol{\Omega})$ by integrating out the variables $\sigma$ as

$$
\exp \left[-\frac{a}{k_{B} T} \sum_{j=1}^{N} f\left(\boldsymbol{\Omega}_{j}\right)\right]=\sum_{\{\sigma\}} e^{-E[\boldsymbol{\Omega}, \sigma] / k_{B} T}
$$

where $k_{B} T$ denotes thermal energy. For uniform helical shape, i.e., $j$-independent strain rates, $\Omega_{2 j}=\Omega_{2}$ and $\Omega_{3 j}=\Omega_{3}$, the summation over $\sigma$ can be performed using transfer matrix method. Assuming periodic boundary condition $\sigma_{1}=\sigma_{N}$, and discarding unimportant constants, 
Discrete elastic model for stretching-induced flagellar polymorphs

$f(\boldsymbol{\Omega})=\frac{A}{2} \Omega_{1}^{2}+\frac{A}{2}\left[\Omega_{2}-\frac{\kappa_{01}+\kappa_{02}}{2}\right]^{2}+\frac{C}{2}\left[\Omega_{3}-\frac{\tau_{01}+\tau_{02}}{2}\right]^{2}-k_{B} T \ln \left[e^{J / k_{B} T} \cosh \Lambda+\sqrt{e^{2 J / k_{B} T} \sinh ^{2} \Lambda+e^{-2 J / k_{B} T}}\right]$,

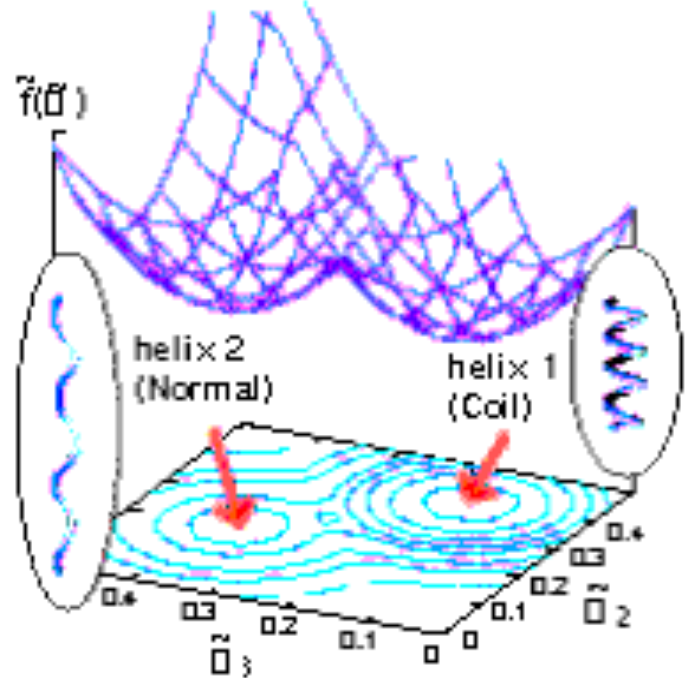

Fig. 1: Plot of the rescaled effective elastic free energy $\tilde{f}=$ $\mathrm{fa} / k_{B} T$, eq. (4), as function of curvature and twist strain rates $\Omega_{2}, \Omega_{3}$ (with $\Omega_{1}=0$ ). The two local minima correspond to the normal and coiled form as indicated. Parameters values are $A /\left(a k_{B} T\right)=1844, C / A=0.8, J / k_{B} T=10$, and $h / k_{B} T=7.7$.

we obtain

$$
\text { see eq. (4) }
$$

where we defined $k_{B} T \Lambda(\boldsymbol{\Omega})=h a+\frac{A a}{2}\left(\kappa_{01}-\kappa_{02}\right)\left[\Omega_{2}-\right.$ $\left.\frac{1}{2}\left(\kappa_{01}+\kappa_{02}\right)\right]+\frac{C a}{2}\left(\tau_{01}-\tau_{02}\right)\left[\Omega_{3}-\frac{1}{2}\left(\tau_{01}+\tau_{02}\right)\right]$ which acts as a strain-dependent external field. The effective elastic energy $f(\boldsymbol{\Omega})$ is plotted in fig. 1 as a function of the two relevant strain rates for one specific parameter set. The potential $f$ is a double-well with two minima corresponding to the normal and the coiled form. Previous theoretic treatments used a very similar shape of the elastic free energy as a starting point [14].

Simulation details - In our hybrid simulations, the energy functional in addition includes a stretching contribution that ensures connectivity of the spheres, $E_{s t}=$ $K / 2 \sum_{j=1}^{N}\left(\left|\mathbf{r}_{j+1}-\mathbf{r}_{j}\right|-a\right)^{2}$. The local elastic translational force $\mathbf{F}_{j}$ and torque about the local tangent $T_{j}$ acting on each sphere are calculated using the variational method described previously [21,22], leading to the coupled Langevin equations:

$$
\partial_{t} \mathbf{r}_{i}=\mu_{0} \mathbf{F}_{i}+\boldsymbol{\xi}_{i}(t), \quad \text { and } \quad \partial_{t} \phi_{i}=\mu_{r} T_{i}+\Xi_{i}(t),
$$

where $\partial_{t}$ is the time derivative. Neglecting hydrodynamic effects, we take the mobility matrix to be diagonal and use the Stokes translational and rotational mobilities of a sphere $\mu_{0}=1 /(3 \pi \eta a)$ and $\mu_{r}=1 /\left(\pi \eta a^{3}\right)$, where $\eta$ is the solvent viscosity. The vectorial random forcings $\boldsymbol{\xi}(t)$ and $\Xi(t)$ model the coupling to a heat bath and obey the fluctuation-dissipation relations. Standard Monte-Carlo Metropolis methods are applied to update $\sigma$. In each Brownian dynamics time step, 20 Monte-Carlo steps are carried out, meaning that equilibration of the $\sigma$ degrees of freedom is much faster than filament shape relaxations.

For the simulations we discretize the Langevin equations (6) with time step $\Delta$ and rescale all lengths, times and energies; the rescaled time step $\tilde{\Delta}=\Delta k_{B} T \mu_{0} / a^{2}$ is for sufficient numerical accuracy chosen in the range $\tilde{\Delta}=10^{-4}$ $10^{-5}$. The stretching modulus is set to $K / k_{B} T a^{2}=10^{4}$ which keeps bond length fluctuations negligibly small. Observables are calculated every $10^{3}-10^{4}$ steps, total simulation times are in the order of $10^{6}-10^{8}$ steps. One filament end is fixed at the origin, and the other end (initially being at its equilibrium position, i.e., $z=L \cos \psi_{1}$ ) is moved along the $\hat{\mathbf{z}}$-axis (identical to the helix axis). We set the maximum extension $z=0.8 \mathrm{~L}$, the total stretching distance is thus $D=\left(0.8-\cos \psi_{1}\right) L$. We fix the number of beads $L / a=N=60$ and the arclength of one helical turn $\ell=15 a$, the pitch angle $\psi$ thus uniquely determines the helical shape of the filament. We set the bend/twist ratio $\Gamma=C / A=0.8$ with $\tilde{A}=A /\left(a k_{B} T\right)=1844$. The bend persistence length is thus $L_{p} / L \simeq 31$, comparable to the experimental conditions $L_{p} / L \simeq 40-120$, obtained from measured values of the bending stiffness $A \simeq 3.5$ $\mathrm{pN} \cdot \mu \mathrm{m}^{2}$ and typical filament length of 7.6-19.5 $\mu \mathrm{m}$ [16]. Although the magnitude of $J$ is experimentally unknown, we assume $J / k_{B} T=10$ sufficiently larger than thermal energy in accord with the observation that thermally assisted nucleation of polymorphic transitions is absent in the stress-free state $[14,15]$. The rescaled stretching speed, $\tilde{V}=V a /\left(\mu_{0} k_{B} T\right)$, is changed in the range $\tilde{V}=0.01-0.2$, to study systematically the rate-dependent elasticity. Assuming $a \simeq 126 \mathrm{~nm}$ (giving $L=a N \simeq 7.6 \mu \mathrm{m}$ for $N=60$ as in the experiment [16]), we obtain $0.3-3.3 \mu \mathrm{m} / \mathrm{s}$ comparable to the experimental $V \simeq 0.4 \mu \mathrm{m} / \mathrm{s}$ [16]. Our filament is thus thicker than a real bacterial flagellar filament by roughly a factor of 5 , which however is not serious as the filament thickness enters hydrodynamic drag coefficients only logarithmically. The pulling end of the filament is free to rotate, i.e., no external torque is applied, while the tethered end is not allowed to rotate, suitable to the experiment condition.

Force-induced shape transformations - In fig. 2, we show one typical force-extension curve obtained at pulling rate $\tilde{V}=0.0124$ (the slowest considered), with several snapshots of the filament at the points specified in 


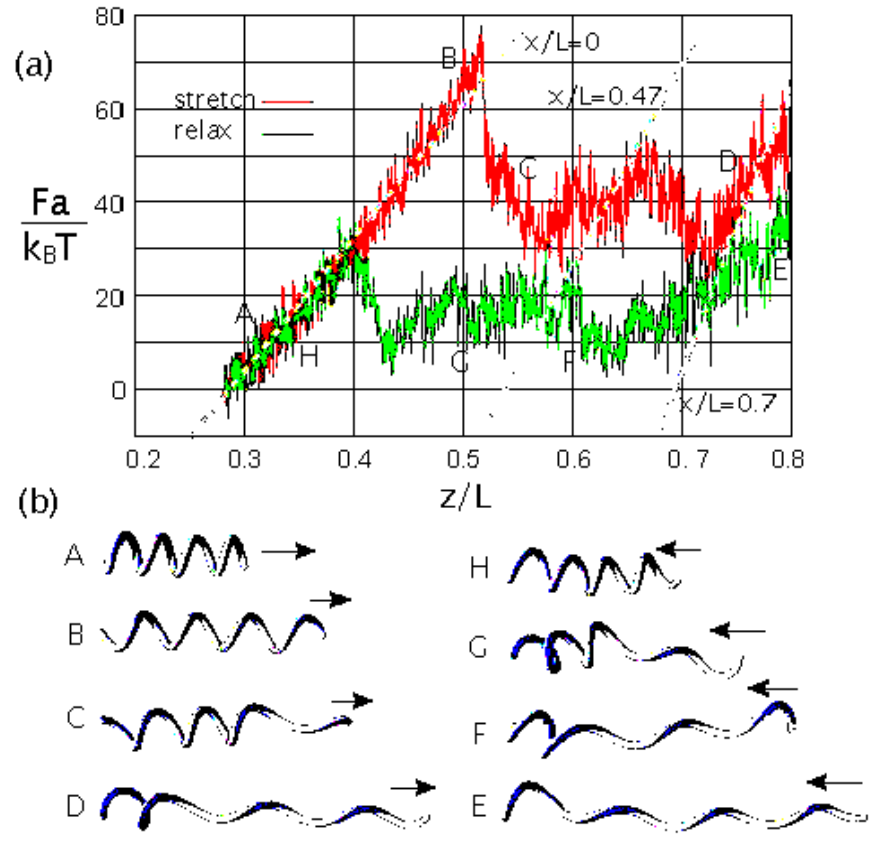

Fig. 2: (a) Typical force-extension curve obtained for $N=60$, $\tilde{A}=1844, C / A=0.8, J / k_{B} T=10$ and $\tilde{h}=h a / k_{B} T=7.7$ at a stretching speed $\tilde{V}=0.0124$. Red line is the stretching, the green one is the relaxing curve. The broken lines follow from the mixed Hookean-spring theory eq. (8) for $x / L=0,0.47$ and 0.7, from left to right. (b) Corresponding snapshots of the filament at points specified in fig. 2 (a).
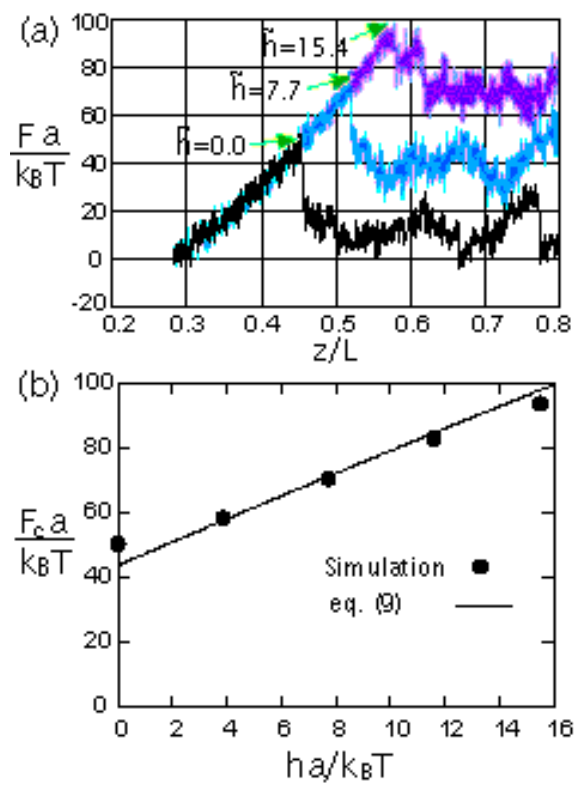

Fig. 3: (a) Stretching force curves for varying chemical potential bias $\tilde{h}=0.0,7.7,15.4$, obtained at $\tilde{V}=0.0124$. All other parameters are the same as in fig. 2 (a). (b) Threshold force, $\tilde{F}_{c}$, obtained from fig. 3 (a), plotted as a function of $\tilde{h}$. Broken line is the analytic result, eq. (9), with $x \simeq 1.32 \ell$ as the best fit. (a). The initial filament shape is coiled, according to the experimental conditions [16]. As the extension $z$ increases, the helix deforms uniformly like a linear spring. Neglecting thermal effects, the restoring force $F$ of a deformed helix is on the linear-elasticity level given by [22]

$$
F=A C \frac{\left(\kappa_{01} \cos \psi-\tau_{01} \sin \psi\right)\left(A \kappa_{01} \sin \psi+C \tau_{01} \cos \psi\right)}{\sin \psi\left(A \sin ^{2} \psi+C \cos ^{2} \psi\right)^{2}}
$$

with $z / L=\cos \psi$. For small forces, Hooke's law, $F \simeq K_{1, L}\left(z-L \cos \psi_{1}\right)$, holds, where $K_{m, L}=$ $\partial F /\left.\partial z\right|_{z=L \cos \psi_{m}}=\left(4 \pi^{2} / \ell^{2}\right)\left(L \sin ^{2} \psi_{m}\right)^{-1}\left(\cos ^{2} \psi_{m} / A+\right.$ $\left.\sin ^{2} \psi_{m} / C\right)^{-1}$ with $m=1,2$, is the linear spring constant $[19,22]$. The spring constant is inversely proportional to the arclength of the helix. At a certain critical force $(\tilde{F} \simeq 70$ in fig. 2 (a)), the uniform helical shape becomes metastable, and a shape transformation occurs from the moving end of the filament via nucleation of the normal form, see the snapshot $\mathrm{C}$ in fig. 2 (b). The filament separates into two domains of different pitch angles $\psi_{1}$ and $\psi_{2}$, and the tension $F$ suddenly drops down to much smaller value; compare B \& C in fig. 2. After the first transition, subsequent more gradual shape transformations at increasing extension $z$ are observed at lower forces. Approximating the filament as two serial Hookean springs of arclength $L-x$ and $x$, we obtain a modified Hooke's law

$$
F=\frac{K_{1, L-x} K_{2, x}}{K_{1, L-x}+K_{2, x}}\left[z-(L-x) \cos \psi_{1}-x \cos \psi_{2}\right] .
$$

In fig. 2 (a), eq. (8) is compared to the numerical data assuming helix compositions of $x / L=0,0.47$ and 0.7 (broken lines) and captures reasonably well the response of the mixed filament. The main point here is that the helical transformation proceeds in subsequent steps.

A normal helical section with pitch angle $\psi_{2}$ appears upon stretching via nucleation. Assuming the helix as a linear spring, the filament elastic energy (relative to its stress-free state) at force $F$ is $E \simeq \frac{1}{2} F^{2} / K_{1, L}$. Once a normal section of arclength $x$ appears, the tensile force drops down to $F^{\prime}$ at constant extension $z$. We note that in the simulations we almost always observe nucleation accompanied by two domain walls. The energy of the mixed state at the transition thus is $E^{\prime} \simeq \frac{1}{2}\left(1 / K_{1, L-x}+1 / K_{2, x}\right) F^{\prime 2}+$ $2 h x+4 J$. Nucleation proceeds only when $E^{\prime}<E$. Using $F^{\prime}=\left(1 / K_{1, L-x}+1 / K_{2, x}\right)^{-1}\left(F / K_{1, L}-\gamma\right)$, where $\gamma=x\left(\cos \psi_{2}-\cos \psi_{1}\right)$, and assuming $K_{1, L-x} / K_{1, L} \simeq 1$ valid for $x / L \ll 1$, we finally obtain the threshold force $F_{c}$ as a function of $x$ and $h$ :

$$
F_{c} \simeq-\gamma K_{2}+\sqrt{\left(K_{1}+K_{2}\right)\left[K_{2} \gamma^{2}+4(h x+2 J)\right]},
$$

where we have used the shorthand notation $K_{1, L-x}=K_{1}$ and $K_{2, x}=K_{2}$.

We perform stretching simulations for five different values of the chemical potential bias, $\tilde{h}$. All other parameters are the same as those in fig. 2 (a). The force-extension 
Discrete elastic model for stretching-induced flagellar polymorphs

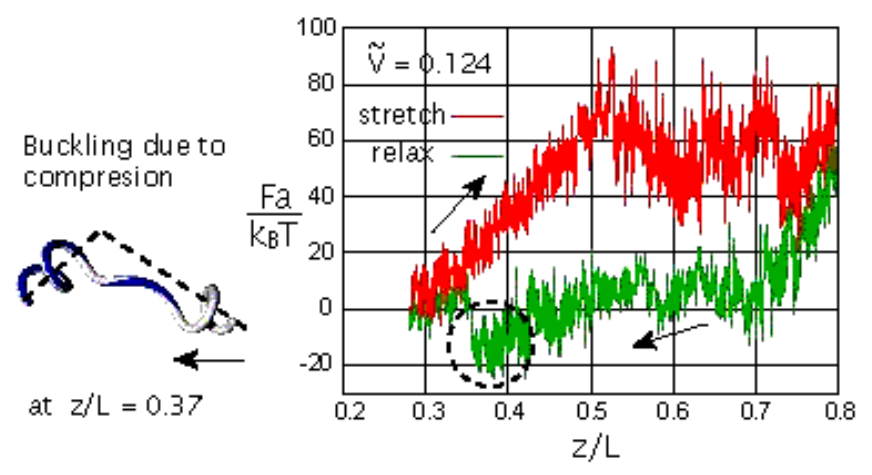

Fig. 4: Force-extension curve at velocity $\tilde{V}=0.124$, ten times faster than fig.2(a). Shown is one stretching (red line) contraction (green) cycle. The snapshot of the buckling state (left) is obtained at stretching $z / L=0.37$ upon contraction and yields negative forces (highlighted by broken circle). All other parameters are the same as used in fig. 2 (a).

curves for $\tilde{h}=0,7.7,15.4$ are shown in fig. 3 (a), from which the critical force $F_{c}$ is obtained as a function of $h$, which is plotted in fig. 3 (b). Since the nucleation domain size $x$ is not determined by our arguments, we fit $x \simeq 19.8 a \simeq 1.32 \ell$ in the comparison of eq. (9) to the numerical data in fig. 3 (b), which is also consistent with the nucleating domain size extracted from the snapshots B and $\mathrm{C}$ in fig. 2(b). The agreement between the numerical data and eq. (9) is excellent, verifying the mixed Hookean spring scaling model.

To demonstrate the experimental relevance of our results, we put experimental values into eq. (9), namely $\psi_{1}=76.7^{\circ}, \psi_{2}=31.3^{\circ}, \ell_{1}=3.4 \mu \mathrm{m}, \ell_{2}=2.5 \mu \mathrm{m}$, $A \simeq 3.5 \mathrm{pN} \mu \mathrm{m}^{2}, L \simeq 7.6 \mu \mathrm{m}[16]$ and $x \simeq 1.32 \ell_{2} \simeq 3.35$ $\mu \mathrm{m}$ from fig. 3 (b). The experimental value of the energy difference between the two polymorphs, $h$, is unknown, and we obtain $F_{c} \simeq 3-5 \mathrm{pN}$ for the range $h \simeq 0.15-0.76$ $\mathrm{pN} \simeq 0.04-0.20 k_{B} T / \mathrm{nm}$. The experimentally determined critical force $F_{c}$ for the coiled-to-normal transition ranges over $3-5 \mathrm{pN}$ [16], quite consistent with our results. The direct (and independent) experimental measurement of $h$ might be hard, but atomistic simulations [24] may in the future check our prediction of $h$.

The contraction process involves discontinuous reverse polymorphic transformations characterized by sudden increases of the tensile force; see fig. 2 (b). The reverse transitions are in general observed at much lower forces and at different extensions, resulting in a large hysteresis.

Kinetic effects - In fig. 4, we show a force-extension curve for the fastest velocity studied by us, $\tilde{V}=0.124$, with all other parameters as in fig. 2 (a). Notable is the negative force in the contraction process at $\tilde{V}=0.124$. This is due to a buckling of the helix via compression; a buckled filament configuration is shown to the left. A buckling-induced negative force has also been observed in the experiment [16].

The domain wall between normal and coiled sections moves in response to the pulling of the filament end. We plot in fig. 罒(a) the domain wall positions $s_{d}$ as a function of the reduced time $V t / D$, where $D$ is the stretching distance defined earlier and thus $D / V$ gives the total stretching time. The domain boundary position is obtained from the discrete $\sigma$-profile along the filament. As noted earlier, we generally observe the occurrence of two domain walls. The trajectories in fig. 5 (a) are strongly asymmetric between the stretch and contraction processes. On the stretching side, $0<V t / D \leq 1$, trajectories for different velocity $V$ collapse. The domain-wall speed $\partial s_{d} / \partial t$ upon stretching approximately follows from a simple geometric argument as

$$
\frac{\partial s_{d}}{\partial t}=\frac{V}{\cos \psi_{2}-\cos \psi_{1}} .
$$

To see this, note that the lengths of the two helices are $s_{d}$ and $L-s_{d}$. Neglecting helix deformations from its intrinsic shape, the end-point position follows as $z \simeq s_{d} \cos \psi_{1}+$ $\left(L-s_{d}\right) \cos \psi_{2}$, which upon inversion yields Eq.(10) where $V=\partial z / \partial t$ is the imposed stretching speed. In the reduced units in fig. 5 (a), this gives the line of slope ( $\cos \psi_{2}-$ $\left.\cos \psi_{1}\right)^{-1}$ independent of $V$, which is shown as a broken line, in reasonable agreement with the numerical data at early times. Deviations at later stages are connected to nonlinear filament deformations.

To quantify rate-dependent dissipation effects, we calculate the work done between $z_{1}=0.3 L$ and $z_{2}=0.8 L$, $\Delta W=\int_{z_{1}}^{z_{2}} d z F$, in both the stretching and contraction processes, which is shown in fig. [5 (b) as a function of $V$. The difference between the two works, which is a measure of dissipation, becomes larger as $V$ increases. Especially for small $V, \Delta W$ is strongly influenced by the stretching rate $V$, consistent with the experimental observations [16]. One trivial contribution to dissipation is due to solvent friction. Assuming the filament to be homogeneously stretched and on the free-draining level (as appropriate for our simulations), the friction work is

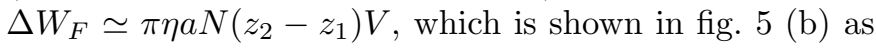
broken lines. As one can see, the solvent friction does not account for the entire dissipation, the difference is due to elastic dissipation within the filament. In the quasi-static limit $V \rightarrow 0, \Delta W$ for stretching and contraction should become equal. Although a direct numerical check of this is difficult because of prohibitively long simulation times, a simple scaling estimate is helpful: Assuming that the helix takes a perfect normal form (all $\sigma=-1$ ) at high stretching $z=0.8 L$ without any elastic deformation, and a perfect coiled form with all $\sigma=1$ at $z=0.3 L$, the total elastic energy change is simply $\Delta E=2 h L$ which determines the work $\Delta W$ in the quasistatic limit at $V=0$. In rescaled units, this leads to $\Delta W / k_{B} T \simeq 2 \tilde{h} N=924$ for

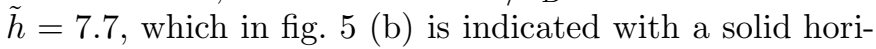
zontal line and is flanked by the stretching and contraction data as expected. In the large $V$ region, i.e. for $\tilde{V}>0.06$ in fig. 5 (b), which probably is beyond experimentally accessible speeds, the force response seems less affected by 


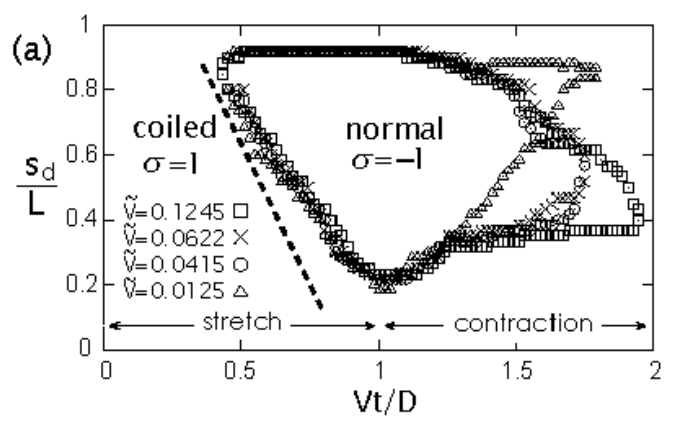

(b)

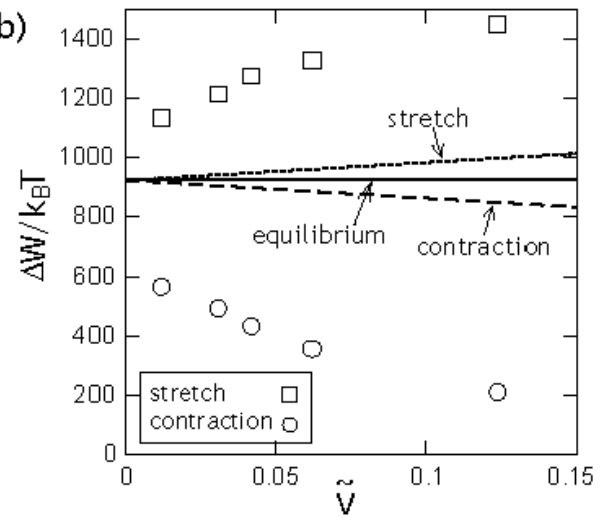

Fig. 5: (a) Trajectories of the rescaled domain-wall positions $s_{d} / L$ as a function of the reduced time $V t / D$ for varying velocities $\tilde{V}$. The slope of the broken line is $\left(\cos \psi_{2}-\cos \psi_{1}\right)^{-1}$ from eq. (10). (b) Mechanical work done between $z_{1}=0.3 L$ and $z_{2}=0.8 L, \Delta W / k_{B} T$, as a function of $\tilde{V}$ in the stretching (square) and contraction (circle) processes. Each data point is the average over three statistically independent runs. (Error bars are smaller than the symbols.) The solid line indicates the equilibrium work, and the broken lines are the friction work for stretching and contraction processes (see text). Parameters are the same as in fig. 2 (a), unless otherwise stated.

the stretching rate $V$ and the apparent rate-dependence is largely due to solvent friction.

Summary - In this letter, we have introduced a bistable helical filament model that accounts for different elastic monomeric states by a discrete Ising-like spin variable along the arclength. Employing hybrid Brownian dynamics Monte-Carlo simulations, we have studied the kinetic effects of uniaxial stretching on the helical shape. The main results are: (i) The helical filament undergoes reversible polymorphic transformations in response to an externally applied uniaxial tension, consistent with the optical-tweezer experiments of Salmonella flagellar filaments. (ii) Experimental force-extension curves including sawtooth pattern and large hysteresis are qualitatively reproduced. (iii) A simple analytic argument based on a mixed Hookean spring model for the critical force of the polymorph induction provides good agreement with numerical and experimental data. (iv) Force-extension curves are strongly pulling rate-dependent, in particular a buckling-induced negative force is observed, all consistent with the experimental observations.
Generalization of our model to include all possible polymorphic flagellar states is straightforward but includes many at present unknown parameters. Applying our model to torque-induced chirality transformations of flagellar filaments, directly relevant to bacterial motility in vivo, is in progress [25].

Financial support from Research Abroad Program of the Japan Society for the Promotion of Science (JSPS) and the German Science Foundation (DFG, SPP1164 and SFB 486) is acknowledged.

\section{REFERENCES}

[1] Berg H. C. and Anderson R. A., Nature (London), 245 (1973) 380.

[2] Darnton N. C. and Turner L. and Rojevsky S. and Berg H. C., J. Bacteriol., 189 (2007) 1756.

[3] Kim M. J. and Powers T. R., Phys. Rev. E, 69 (2004) 061910.

[4] Reinchert M. and Stark H., Eur. Phys. J. E, 17 (2005) 493.

[5] Macnab R. M. and Ornston M. K., J. Mol. Biol., 112 (1977) 1.

[6] Namba K. and Vonderviszt F., Q. Rev. Biophys., 30 (1997) 1.

[7] Asakura S., Adv. Biophys. (Japan), 1 (1970) 99.

[8] Yamashita I. et al., Nat. Struct. Biol., 5 (1998) 125.

[9] Calladine C. R., Nature (London), 255 (1975) 121.

[10] Srigiriraju S. V. and Powers T. R., Phys. Rev. Lett., 94 (2005) 248101.

[11] Turner L., Ryu W., and Berg H., J. Bacteriol., 182 (2000) 2793.

[12] Hotani. H., J. Mol. Biol., 156 (1982) 791.

[13] Washizu M., Shikida M., Aizawa S., and Hotani H., IEEE Trans. Industry Applications, 28 (1992) 1194.

[14] Coombs D., Huber G., Kessler J. O., and Goldstein R. E., Phys. Rev. Lett., 89 (2002) 118102.

[15] Goldstein R. E., Goriely A., Huber G., and WolGemuth C. W., Phys. Rev. Lett., 84 (2000) 1631.

[16] Darnton N. C. and Berg H. C., Biophys. J., 92 (2007) 2230.

[17] Zhao J. M., Kim M., Scherer N. F. and Cluzel P., unpublished.

[18] Arkhipov A., Freddolino P.L., Imada K., Namba K. and Schulten K., Biophys. J., 91 (2006) 4589.

[19] Love A. E. H, A Treatise on the Mathematical Theory of Elasticity (Dover, New York) 1944.

[20] Zimm B. H. and Bragg J., J. Chem. Phys., 31 (1956) 526.

[21] Chirico, G. and Langowski J., Biopolymers, 34 (1994) 415.

[22] WAda H. and Netz R. R., Europhys. Lett., 77 (2007) 68001.

[23] Smith B., Zastavker Y. V., and Benedek G. B., Phys. Rev. Lett., 87 (2001) 278101.

[24] Kitao A. et al., Proc. Nat. Acad. Sci. U.S.A., 103 (2006) 4894.

[25] WAda H. and Netz R. R., manuscript in preparation (2008). 